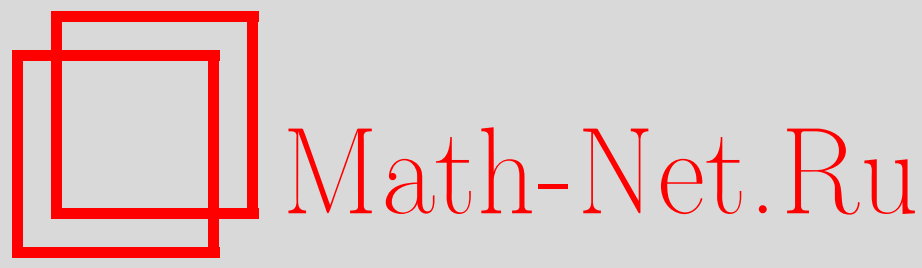

В. П. Маслов, Критические показатели как следствие винеровского квантования термодинамики, ТМФ, 2012, том 170, номер 3, 457-467

DOI: https://doi.org/10.4213/tmf6778

Использование Общероссийского математического портала Math-Net.Ru подразумевает, что вы прочитали и согласны с пользовательским соглашением http://www.mathnet.ru/rus/agreement

Параметры загрузки:

IP: 34.229 .108 .108

26 апреля 2023 г., 17:50:03

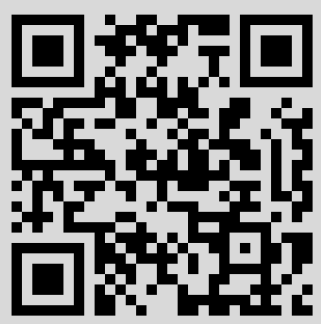




\section{КРИТИЧЕСКИЕ ПОКАЗАТЕЛИ КАК СЛЕДСТВИЕ ВИНЕРОВСКОГО КВАНТОВАНИЯ ТЕРМОДИНАМИКИ}

\footnotetext{
Построено семейство идеальных газов, зависящих от критического значения фактора сжимаемости $Z$ для чистых газов. Показано, что критические индексы реальных простых жидкостей, как и многие другие термодинамические эффекты, легко и естественно следуют из концепции винеровского квантования современной термодинамики.
}

Ключевые слова: туннельный канонический оператор, критические индексы, квантование термодинамики.

\section{Посвящается памяти дорогого Алеко ${ }^{1)}$}

\section{1. ФЛУКТУАЦИИ И СЕМЕЙСТВО ИДЕАЛЬНЫХ ГАЗОВ}

Крупнейший математик XIX века Л. Кронекер сказал: “Бог создал натуральный ряд, все остальное дело рук человека". В работе "О догмате натурального ряда" П. К. Рашевский пишет: "Знаменитые отрицательные результаты Гёделя 30-х годов в своем фундаменте исходят из убеждения: сколько бы ни продолжать построение математических формул для данной (полностью формализованной) математической теории, принципы пересчета и упорядочения формул остаются обычными, т. е. подчиненными схеме натурального ряда. Разумеется, это убеждение даже не оговаривалось, - настолько оно считалось очевидным".

Напомню, что "отрицательные результаты" Гёделя (невозможность формализовать арифметику) подорвали фундамент, на котором основывалась программа Гильберта о построении всей математики в виде полностью формализованной теории. Действительно, из теоремы Гёделя о неполноте вытекает, что любая формальная система, включающая в себя догмат натурального ряда (т. е. его обычную аксиоматику), принципиально порочна: если она непротиворечива, то она неполна, ибо содержит истинные арифметические утверждения, которые нельзя ни доказать, ни опровергнуть. Анализ построений великого Гёделя показывает, что эти арифметические утверждения касаются очень больших чисел - эти числа можно формально определить, но реально вычислить их никак нельзя. Гильберт, как известно,

1) См. январский и февральский выпуски журнала, посвященные А. Н. Тахвелидзе.

* Московский государственный университет им. М. В. Ломоносова, Москва, Россия. E-mail: v.p.maslov@mail.ru 
после анализа этой теории математической логики Гёделя довольно долго не мог оправиться от депрессии. Автор, напротив, полагает, что теорема Гёделя приводит к объяснению ряда парадоксов термодинамики при привлечении дополнительного инструмента, например измерительного прибора. Приведем элементарные примеры.

Еще в IV веке до нашей эры греческий философ Эвбулид из Милета сформулировал парадокс кучи: начиная с какого количества песчинок можно назвать их совокупность кучей? Например, при покупке 1 кг сахарного песка точный подсчет числа сахарных крупинок не только затруднителен, но и не имеет практического смысла. Однако "кучу" 1 кг сахарного песка можно разделить на две кучи по $1 / 2$ кг или на кучи по 100 г. Операции сложения и умножения для них остаются. Иначе говоря, для куч арифметические правила сохраняются. Иначе говоря, для куч арифметические правила сохраняются. Масштаб определяется измерительным прибором (например, весами с гирями). Если убирать по одной песчинке, говорил Эвбулид из Милета, то мы придем к небольшому числу песчинок. А эта совокупность - уже не куча.

По-видимому, утверждение, что если при перестановке двух крупинок понятие кучи не меняется, так же как не меняется правило арифметики куч, то это и есть основное свойство этого понятия: в куче теряется индивидуальность крупинки. С точки зрения понятий статистической физики это уже переход от статистики Больцмана (энтропии Больцмана, равной энтропии Шеннона) к статистике типа статистики Бозе-Эйнштейна, хотя крупинки различимы между собой.

Мы хотим сказать заранее, что к этим глубочайшим логическим проблемам имеет отношение равновесная термодинамика.

Уже во второй половине ХХ века делались попытки произвести реформу числового ряда с точки зрения "нечетких множеств", "интервального анализа", "нестандартного анализа" и т. п. Если говорить на языке физиков, то это означает необходимость учета флуктуаций.

Чтобы продемонстрировать разницу между равновесной и неравновесной термодинамиками, рассмотрим простой пример стакана горячего чая, в котором мы размешали ложку сахара. Не так давно в русском языке вошло в обиход слово "устаканиться". Мы дождемся, когда жидкость в стакане не будет вращаться устаканится, но чай еще будет горячим. Это означает, что мы допускаем небольшие флуктуации жидкости, иначе чай остынет. Чем меньше флуктуации мы допускаем, тем холоднее будет чай и тем больше времени мы будем ждать, чтобы приступить к чаепитию. Если флуктуации с нашей точки зрения уже допустимы, мы говорим, что жидкость “устаканилась". Этот процесс совершенно аналогичен переходу от неравновесной системы (термодинамики) к равновесной, в которой допустимы сравнительно небольшие флуктуации.

Можно изменить концепцию Кронекера, например, следующим образом: Бог создал воздуx, а следовательно, числа Авагадро и Лошмидта, т. е. величины от $10^{19}$ до $10^{23}$, а также неизбежные флуктуации воздуха. Отсюда следует невозможность практически точно определить такого рода величины.

Шредингер в своей знаменитой работе "Что такое жизнь с точки зрения физики?" высказывает гипотезу, что если число частиц $N$ достаточно велико, то мы не можем рассчитывать получить результат более точный, чем с точностью до $\sqrt{N}$. Это означает, что Шредингер предполагает возможность флуктуаций порядка $\sqrt{N}$. Причем он назвал это утверждение "законом природы". Более точно математически это можно было бы выразить так: вероятность отклонения числа частиц $N$ на вели- 
чину, большую чем $\sqrt{N}$, достаточно мала ${ }^{2)}$. Мы называем это "мягким множеством" из $N$ элементов. "Закон природы” Шредингера противоречит догмату натурального ряда.

Если мы дополним принцип догмата натурального ряда внешним прибором, но не откажемся от арифметики, т. е. от коммутативности сложения, то это значит, что мы считаем с некоторой заданной точностью, и если учесть соображения вероятности, то с "мягкой" заданной точностью. Эта мягкая точность позволяет, нумеруя, сосчитать все элементы конечного множества. Мы лишь говорим, что если неточный подсчет отличается от точного на данную величину, то мы пренебрегаем этой неточностью. В так называемом законе равнораспределения участвуют лишь небольшие целые числа. Этот закон тоже претерпевает изменения. А это уже затрагивает привычную философию, укоренившуюся веками и может вызвать протесты не только естественников, но и философов.

Прогрессивные российские гуманитарии категорически отвергали геометрию Лобачевского (см. Н. Чернышевский "Письма к детям"). Отметим, что геометрия Лобачевского в действительности есть однопараметрическое семейство геометрий, зависящее от радиуса (кривизны) и при радиусе, стремящемся к бесконечности, переходящее к евклидовой геометрии.

Советские философы подвергали острокизму последователей принципа дополнительности Бора, который он почерпнул из биологии и психологии. Вместе с тем, если принять этот принцип дополнительности как “дополнение" к новой концепции числа частиц $N$ с возможными флуктуациями порядка $\sqrt{N}$, то мы придем к понятию химическиого потенциала $\mu$ и положению, аналогичному принципу неопределенности Гейзенберга: чем меньше флуктуации $\mu$, тем больше флуктуации $N$.

Прежде всего давление $P$ поршнем уменьшает объем $V$ воздуха в сосуде - принцип дополнительности Бора связывает эти две субстанции. Увеличение температуры $T$ увеличивает хаотическую скорость частиц, т. е. увеличивает хаос, определяемый энтропией. Эти две величины отвечают принципу дополнительности Бора.

Принцип дополнительности Бора связан с принципом неопределенности Гейзенберга - уменьшение флуктуации одной компоненты соответствует увеличению флуктуации дополнительной субстанции. Принцип дополнительности Бора развивали и применяли не только такие физики широких взглядов на мир, ка Борн, но и такие прагматики, как Паули. По принципу дополнительности сопоставлялись, например, движение и покой. Но особенно существенную роль принцип дополнительности играет в равновесной и неравновесной термодинамиках. Бор объясняет этот принцип вмешательством измерительного прибора.

В нашем примере с сахарным песком измерительный прибор - весы - также играет свою роль, особенно если в процессе взвешивания имеет место "раструска" (подобно измерению температуры ртутным термометром, которое отбирает часть энергии (тепла) у окружающих частиц).

Точно так же, как существует дополнительность "время-энергия", в термодинамике существует дополнительность "время наблюдения - величина флуктуации", поскольку к состоянию равновесной термодинамики мы приходим из состояния неравновесной термодинамики, как это было показано в примере "устаканивания". Здесь существенную роль играет вязкость, торможение, которое и приводит к равновесной термодинамике с точностъю до определенной величины флуктуации. Старая

2) Между тем эта работа Шредингера сыграла, как известно, существенную роль в теории генов. 
термодинамика была основана на статистике Больцмана, а последняя - на догмате натурального ряда. Но оказалось, что догмат натурального ряда столь же непригоден для совокупностей таких больших масштабов в термодинамике, как евклидова геометрия для масштабов Вселенной.

Мы построим ниже однопараметрическое семейство идеальных газов для описания совокупностей таких масштабов.

ПримеР 1. Приведем пример знаменитой теоремы теории чисел Эрдоша - решение старинной задачи, имеющей латинское название "partitio numerorum". В этой задаче участвует целое число $M$, которое разлагается на $N$ слагаемых, например $M=5, N=2: 5=1+4=2+3$, что дает два варианта $\mathcal{M}$ решения этой задачи: $\mathcal{M}=2$.

Если $M=10^{23}, N=1$, то вариант разложения всего один: $\mathcal{M}=1$. Если $M=10^{23}$ и $N=10^{23}$, то вариант разложения также только один - это сумма единиц, т. е. $\mathcal{M}=1$. Следовательно, найдется такое число $N_{\text {c }}$, при котором число вариантов разложения $\mathcal{M}$ будет максимально (это число, вообе говоря, не единственно). Величина $\log _{2} \mathcal{M}$ называется энтропией Хартли. В точке, где она достигает своего максимума, возникает максимум энтропии. Эта задача о числе вариантов $\mathcal{M}$, как известно, сводится к числу решений $\mathcal{M}$ системы диофантовых уравнений

$$
\sum_{i=0}^{\infty} N_{i}=N, \quad \sum_{i=0}^{\infty} i N_{i}=M .
$$

Неединственность указанного выше максимума и неопределенность числа этих максимумов позволили Эрдошу получить результат лишь с точностью до $o(\sqrt{M})$. Тем самым он отступил от догмата натурального ряда в направлении мягкого множества ${ }^{3)}$.

Соотношения (1) соответствуют физическим соотношениям вида (общий случай см. в работе [1])

$$
\sum_{i=0}^{\infty} N_{i}=N, \quad \sum_{i=0}^{\infty} \varepsilon_{i} N_{i}=\mathcal{E},
$$

где $N_{i}$ - число частиц на $i$-м уровне энергии, $\varepsilon_{i}$ - дискретные наборы энергий, $\mathcal{E}-$ энергия.

Поскольку попытки перейти к числам, большим $10^{15}$, в современных многопроцессорных ЭВМ связывают с увеличением пространственной размерности, мы применяем здесь подход, связанный с размерностью пространства (в том числе и с дробной - фрактальной размерностью).

Построим однопараметрическое семейство идеальных газов согласно концепции мягкого множества. Если рассматривается частный нерелятивистский случай, когда гамильтониан системы $H=p^{2} / 2 m$, где $p$ - импульс, то задаче (1) с точностью до постоянных множителей соответствует двумерный случай.

Ландау и Лифшиц [2] дают примечание относительно "импульсного пространства": "Явление накапливания частиц в состоянии с $\varepsilon=0$ часто называют конденсацией Бозе-Эйнштейна. Подчеркнем, что речь может при этом идти разве что о “конденсации в импульсном пространстве"; никакой реальной конденсации в газе,

${ }^{3)}$ Главный член $N_{\mathrm{cr}}$, при котором число решений $\mathcal{M}$ системы (1) максимально, имеет вид $c \sqrt{M} \ln M$, где $c$ - строго определенная константа. 
конечно, (!) не происходит". Мы же покажем, что реальная конденсация в обычном газе происходит.

Энергия $\mathcal{E}$ (т. е. исходное число $M$ в задаче partitio numerorum) при $\gamma=(d-1) / 2$, где $d$ - "фрактальная" нецелая размерность, $d=D / 2$, имеет вид

$$
\begin{aligned}
\mathcal{E} & =\frac{T^{2+\gamma}}{\Lambda^{1+\gamma} \Gamma(2+\gamma)} \int_{0}^{\infty} \xi^{1+\gamma}\left\{\frac{1}{e^{(\xi-\kappa)}-1}\right\} d \xi= \\
& =\frac{1}{\Lambda^{1+\gamma}} T^{2+\gamma} \operatorname{Li}_{\gamma+2}\left(e^{\mu / T}\right),
\end{aligned}
$$

где $\kappa=\mu / T, \operatorname{Li}_{\gamma}(\cdot)$ - полилогарифм: $\operatorname{Li}_{\gamma+2}(1)=\zeta(\gamma+2), \zeta$ - дзета-функция Римана, $\Lambda$ - некоторая константа. Теперь мы можем точно математически сформулировать основной принцип термодинамики, отвечающий приблизительному сохранению плотности газа (это отвечает формулировке физиков в равновесной термодинамике: "плотность однородна в сосуде").

ЗАмЕчАниЕ 1. Из этого всем очевидного факта строго следует новое соотношение для идеального газа, отдельное для каждой молекулы чистого газа. Это следует из достаточно простой теоремы теории чисел.

ЗАмечание 2. Отношение $M / k N T$ "безразмерно" с учетом константы Больцмана $k$ в том смысле, что в физике $M$ соответствует энергии $\mathcal{E}$. Величина $Z=\mathcal{E} / R T$, где $R$ - газовая постоянная, называется фактором сжимаемости.

Отсюда и из (3) вытекает следствие.

СлЕДСТВИЕ 1. При $\mu=0$ возникают максимумы $\mathcal{M}$ и энтропии. Мы получаем однопараметрическое (параметр $\gamma$ ) семейство максимальных значений критических факторов сжимаемости

$$
Z_{\text {degen }}=\left.\frac{M}{N T}\right|_{\mu=0}=\frac{\zeta(\gamma+2)}{\zeta(\gamma+1)} .
$$

Будем называть “бозе-газом" (в кавычках) газ, описываемый соотношением (3) при любом $\gamma$.

Отметим, что дробная размерность (или фрактальная размерность) в пространстве импульсов не имеет особого физического смысла. Это понятие может быть удобно для сокращения записи, что крайне важно в теории передачи информации. На самом деле дробная размерность характеризует лишь плотность спектра (по терминологии Ландау и Лифшица количество собственных значений, которые "помещаются" в одной ячейке). Грубо говоря, спектр данной молекулы и физически может быть связан с числом "активных" степеней свободы, целое число которых, как мы видели, изменяется, когда применяется "реформа натурального ряда".

На рис. 1 приведены графики из работы [3], в которых совпадение с новым идеальным "бозе-газом" имеет место вплоть до критической точки $T=T_{\text {с на }}(P, V)$-диаграмме, т. е. при давлениях, в три-четыре раза превышающих точность совпадения со старым идеальным газом $P V=R T$.

\section{2. ВИНЕРОВСКОЕ (ТУННЕЛЬНОЕ) КВАНТОВАНИЕ}

При винеровском квантовании ${ }^{4}$ [4], [5] в качестве основных операторов берутся не оператор импульса $\widehat{p}=i h \frac{\partial}{\partial x}$ и оператор умножения на $x$, а оператор $\widehat{D}=\varepsilon \frac{\partial}{\partial x}$,

4) В работах [4], [5] оно названо туннельным квантованием. 


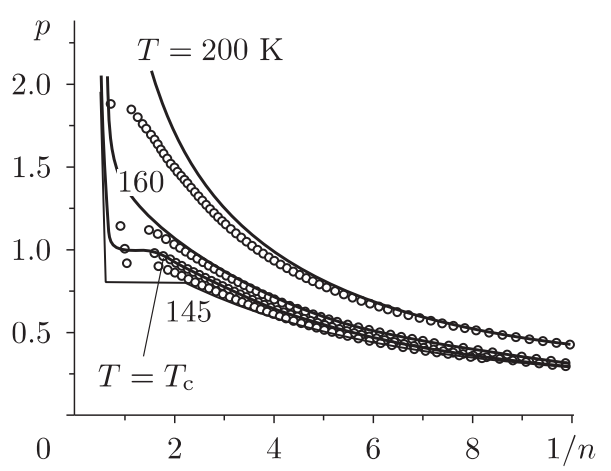

$\mathrm{a}$

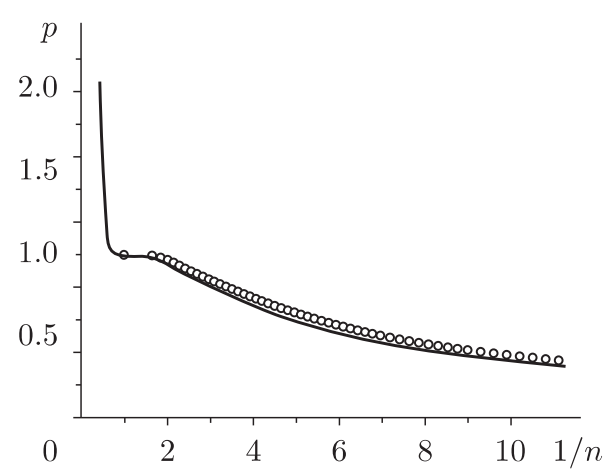

б

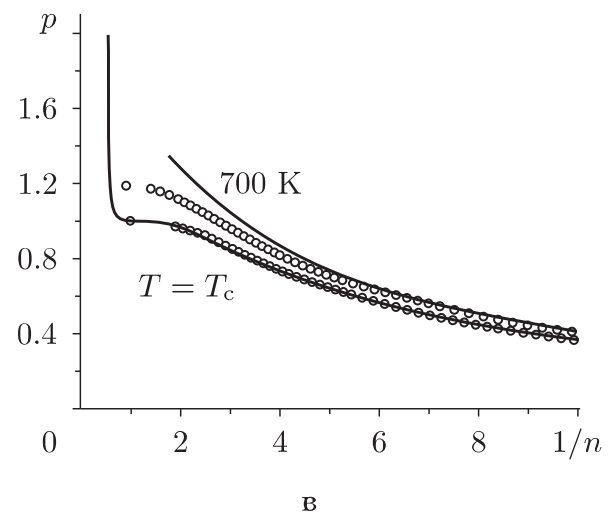

Рис. 1. Изотермы для аргона, $Z_{\mathrm{cr}}=\zeta(\gamma+2) / \zeta(\gamma+1)=0.29$ (а), воды, $Z_{\text {cr }}=0.23$ (б) и меди, $Z_{\text {cr }}=0.39$ (в). Сплошные линии соответствуют экспериментальным данным. Линии, образованные кружочками, построены в соответствии с изотермой идеального бозе-газа.

где $D=\partial / \partial x$ - оператор Хевисайда, и оператор умножения на $x$. Константу $\varepsilon$ мы определили ранее [6] как вязкость. В отличие от константы Планка, константа $\varepsilon$ может плавно зависеть от переменных системы. Главное отличие винеровского квантования от евклидова и обычного состоит в том, что оно рассматривается с точностью до $O\left(\varepsilon^{k}\right)$, где $k$ - некоторое фиксированное число, т. е. факторизованное по $O\left(\varepsilon^{k}\right)$. Но прежде всего нужно определить пространство, в котором эти операторы действуют, и доказать для них принцип неопределенности.

Как известно, оператор Хевисайда связан с двусторонним преобразованием Лапласа. Это показано еще Ван-дер-Полем и Бреммером [7]. Определим набор функций $\varphi(p)$, к которым мы применяем двустороннее преобразование Лапласа, именно

$$
\varphi(p)=\int_{0}^{\infty} e^{-p^{2} \xi} \Xi(\xi) d \xi,
$$

так что эти функции сами являются односторонним преобразованием Лапласа

$$
\widehat{F}_{\lambda} \Xi(\xi)=\int_{0}^{\infty} e^{-\lambda \xi} \Xi(\xi) d \xi
$$


при $\lambda=p^{2}$. Через $\widehat{F}_{\lambda}^{ \pm}$будем обозначать оператор двустороннего преобразования Лапласа

$$
\widehat{F}_{\lambda}^{ \pm} \varphi(p)=\int_{-\infty}^{\infty} e^{-\lambda p} \varphi(p) d p .
$$

Если функции $\Xi(\xi)$ финитные бесконечно дифференцируемые, то замыкание по такой области определения оператора $D$ можно проводить в пространстве Бергмана. Функции $\Psi(x)=\widehat{F}_{x}^{ \pm} \varphi(p)$ у нас будут аналогичны $\Psi$-функциям в квантовании Шредингера. При этом $\Psi^{*}(x)=\Psi(x)$, поскольку эти функции действительнозначные.

Прежде всего отметим, что квадрат функции или, что то же самое, квадрат дисперсии $\Delta \widehat{f}$ оператора $\widehat{f}$ есть $(\Delta \widehat{f})^{2}=\left|(\widehat{f}-\bar{f})^{2}\right|$, поскольку оператор $\widehat{f}$ несамосопряженный, то $(\widehat{f}-\bar{f})^{2}$ не обязательно является положительной функцией, и, следовательно, нужно брать ее по модулю. Поэтому соответствующая теорема для общих операторов, вообще говоря, неверна. Но для операторов $\widehat{D}=\varepsilon D$ и $x$ на приведенном пространстве функций мы получим

$$
|\Delta \widehat{D}||\Delta x| \geqslant \frac{\varepsilon}{2}
$$

Нетрудно убедиться, что доказательство Вейля, приведенное в примечании к $\S 16$ главы II книги [8], без труда переносится на операторы $\widehat{D}$ и $x$ в указанном пространстве функций.

Применим винеровское квантование к термодинамике. Термодинамический потенциал $G=\mu N$ является действием $\mathbb{S}=\int p d q$ на двумерном лагранжевом многообразии $\Lambda^{2}$ в четырехмерном фазовом пространстве $q_{1}, q_{2}, p_{1}, p_{2}$, где $q_{1}$ и $q_{2}$ - соответственно давление $P$ и температура $T, p_{1}$ равно объему $V$, а $p_{2}$ равно энтропии $S$ с обратным знаком. Все другие потенциалы (внутренняя энергия $E$, свободная энергия $F$ и энтальпия $W$ ) суть результаты проецирования лагранжева многообразия на координатные плоскости $p_{1}, p_{2}$ :

$$
\begin{gathered}
E=-\int \vec{q} d \vec{p}, \quad \vec{q}=\left\{q_{1}, q_{2}\right\}, \quad \vec{p}=\left\{p_{1}, p_{2}\right\}, \\
W=-\int\left(q_{2} d p_{2}+q_{1} d p_{1}\right), \quad F=\int\left(q_{1} d p_{1}-q_{2} d p_{2}\right) .
\end{gathered}
$$

При винеровском квантовании имеем

$$
N=\varepsilon \frac{\partial}{\partial \mu}, \quad V=\varepsilon \frac{\partial}{\partial p}, \quad S=-\varepsilon \frac{\partial}{\partial T} .
$$

Следовательно, роль времени $t$ при квантовании будет играть $\ln (-\mu)$ :

$$
G=\mu N \sim \varepsilon \mu \frac{\partial}{\partial \mu}=\varepsilon \frac{\partial}{\partial \ln \mu} .
$$

Заметим, что винеровское квантование уравнения Ван-дер-Ваальса (ВДВ) при $\varepsilon \rightarrow 0$ дает правило Максвелла (см. ниже).

Как мы увидим ниже, критическая точка и точки спинодали являются фокальными и поэтому при $\varepsilon \rightarrow 0$ не переходят в "классику", т. е. в модель ВДВ. Точки спинодали, подобно точкам поворота в квантовой механике, приближаются функцией Эйри, а критическая точка - точка возникновения двух точек поворота (двух функций Эйри) - приближается функцией Вебера (см. [9]). Именно через функцию Вебера выражается в уравнении Бюргерса точка возникновения ударной волны при $\varepsilon \rightarrow 0$. Если вне этих точек совершить переход при $\varepsilon \rightarrow 0$, то получится модель ВДВ-Максвелла. Однако в самих этих точках предельный переход нарушается. 
Функция Вебера дает для решения $v$ уравнения Бюргерса величину порядка $\sqrt[4]{\varepsilon}$. Поэтому так называемые "классические" критические показатели Ландау [2] оказываются грубо не совпадающими с экспериментом. Функция Вебера дает особенности вида $\varepsilon^{-1 / 4}$, а функция Эйри - особенность вида $\varepsilon^{-1 / 6}$.

Приведем более подробное рассмотрение уравнения Бюргерса

$$
\frac{\partial v}{\partial t}+v \frac{\partial v}{\partial x}-\frac{\varepsilon}{2} \frac{\partial^{2} v}{\partial x^{2}}=0,\left.\quad v\right|_{t=0}=p_{0}(x),
$$

его решение при $\varepsilon \rightarrow 0$ будем называть (обобщенным) решением уравнения простой волны

$$
\frac{\partial p}{\partial t}+p \frac{\partial p}{\partial x}=0,\left.\quad p\right|_{t=0}=p_{0}(x),
$$

функцию $p_{\text {об }}=\lim _{h \rightarrow 0} v$ (волны Римана).

Решение $v$ уравнения Бюргерса выражается через логарифмическую производную

решения $u$ уравнения теплопроводности

$$
v=-\varepsilon \frac{\partial}{\partial x} \ln u
$$

$$
\frac{\partial u}{\partial t}=\frac{\varepsilon}{2} \frac{\partial^{2} u}{\partial x^{2}},\left.\quad u\right|_{t=0}=\exp \left\{-\frac{1}{\varepsilon} \int_{-\infty}^{x} p_{0}(x) d x\right\} .
$$

Таким образом, исходная задача сводится к изучению логарифмического предела решения уравнения теплопроводности. Известно, что решение задачи (9) имеет вид

$$
u=(2 \pi \varepsilon t)^{-1 / 2} \int_{-\infty}^{\infty} \exp \left\{-\frac{1}{2 t h}\left((x-\xi)^{2}+2 t \int_{-\infty}^{\xi} p_{0}(\xi) d \xi\right)\right\} d \xi
$$

Вне фокальных точек асимптотика интеграла (10) вычисляется методом Лапласа, при $t<t_{\mathrm{cr}}\left(t_{\mathrm{cr}}-\right.$ точка возникновения ударной волны) она имеет вид

$$
u=\left(|J(\xi(x, t), t)|^{-1 / 2}+O(\varepsilon)\right) e^{-S(x, t) / \varepsilon},
$$

где $J$ - якобиан, а $S(x, t)$ - “действие". При $t>t_{\text {сr }}$ существуют три точки $r_{1}(x)$, $r_{2}(x), r_{3}(x)$ на кривой $\Lambda^{t}$, проекции которых на ось $x$ совпадают, или, иначе говоря, уравнение $Q(t, \xi)=x$ при $x \in\left(x_{1}, x_{2}\right)$ имеет три решения $\xi_{1}(x, t), \xi_{2}(x, t), \xi_{3}(x, t)$.

Обозначим $S(x, t)=\int_{-\infty}^{r(x)} p d x$ при $x<x_{1}, x>x_{2}$ и $S(x, t)=\min \left(S_{1}, S_{2}, S_{3}\right)$, $S_{j}=\int_{-\infty}^{r_{j}(x)} p d x$, где $j=1,2,3$, при $x \in\left[x_{1}, x_{2}\right]$.

Приведенные рассуждения позволяют получить обобщенное разрывное решение задачи (7) при временах $t>t_{\mathrm{cr}}$. Оно определяется функцией $p=p(x, t)$, задающей существенные области кривой $\Lambda^{t}$. Заметим, что отсюда, в частности, следует известное в гидродинамике правило равных площадей для определения фронта ударной волны, эволюция которой описывается уравнением (7). Отметим, что это в точности отвечает правилу Максвелла для уравнения ВДВ.

При зарождении ударной волны возникает одномерное лагранжево многообразие $x=p^{3}$. Для преобразования Лапласа это означает, что если мы находимся в области, где применим асимптотический метод Лапласа, т. е. где

$$
u(x)=\frac{1}{\sqrt{\varepsilon}} \int_{0}^{\infty} e^{-(p x+\widetilde{S}(p)) / \varepsilon} d p, \quad \lim _{p \rightarrow 0} \frac{\widetilde{S}(p)}{p^{4}}<\infty,\left.\quad \widetilde{S}^{(4)}(p)\right|_{p=0} \neq 0,
$$

и $\partial^{2} \widetilde{S} / \partial p^{2} \neq 0$ в точке $\partial \widetilde{S} / \partial p=x$, то в этом случае интеграл (12) при $\varepsilon \rightarrow 0$ ограничен. При $x=0$ интеграл (12) выражается через функцию Вебера, и, следовательно, решение уравнения Бюргерса $v\left(\right.$ см. (8)) имеет нуль порядка $\varepsilon^{1 / 4}$ при $\varepsilon \rightarrow 0$. Чтобы 
решение имело нуль порядка $\varepsilon^{1 / 4}$ в нефокальной точке, мы должны проинтегрировать его по $x$ дробным оператором $D^{-1 / 4}$. Применение оператора $D^{-1 / 4}$ к единице дает приблизительно $x^{1 / 4}$.

В случае термодинамики роль $x$ играет давление $P$, а роль импульса $p$ играет объем $V$. Поэтому $V \sim P^{1 / 4}$, т. е. $P_{\text {cr }} \sim\left(V-V_{\text {cr }}\right)^{4} \sim\left(\left(\rho-\rho_{\text {cr }}\right) R / \rho \rho_{\text {cr }}\right)^{4}$.

Вслед за Грином [10] глубокий экспериментатор Иванов [11] задает вопрос: почему отклонения от классической теории в критической опалесценции наблюдаются в пределах сотых градуса от критической точки, тогда как отклонения в термодинамических свойствах показывают неклассическое поведение много дальше от нее?

Допускается ли в принципе объяснение в предъявленной автором концепции экспериментального значения критического индекса 4.3 ?

Если в классической механике нет зависимости гамильтониана от постоянной Планка $\hbar$, то в классической термодинамике есть медленная зависимость вязкости от значений $T$ и $\rho$, а значит, и наоборот, если мы формально обратим эту зависимость. В нашей картине (см. рис. 7 и рис. 8 в работе [12]) "растяжение" $P_{\text {cr }}, T_{\text {cr на }}$ эксперименте у реальных газов большее, чем в модели ВДВ, что позволяет ввести в "растяжение" параметр $\varepsilon^{0.07}$ (т. е. $P=\varepsilon^{0.07} V^{3}$ ) и получить индекс $\delta=4.3$. Так что, вообще говоря, это возможно.

В силу вышесказанного новые критические индексы возникают только благодаря квантованию сопряженных пар $\{P, V\},\{T, S\}$. Значит, соотношения между интенсивными величинами можно брать классические, поскольку все делается в предположении бесконечно малой вязкости. В этом случае можно переходить и к другим координатам - давлению и плотности. Обозначив, как это принято, $p=\left(P-P_{\text {cr }}\right) / P_{\text {cr }}, \theta=\left(T-T_{\text {cr }}\right) / T_{\text {cr }}, v=\left(\rho-\rho_{\text {cr }}\right) / \rho_{\text {cr }}$, имеем в классическом случае ([13], с. 344) $p \sim v^{3}, v \sim \theta^{1 / 2}, \theta \sim v^{2}$. В классическом случае получаем $p \sim \theta^{3 / 2}$. При винеровском квантовании мы получим $\beta=0.375$ (ср. [13], с. 356) в пределе при $\varepsilon \rightarrow 0$, а значит, неточно (без учета растяжения). Это получается для проквантованного по Винеру уравнения ВДВ.

В монографии [2] отмечается специфическая особенность фотонного газа: число частиц $N$ в нем является переменной величиной, а не заданной постоянной, как в обычном газе. Значит, поскольку в термодинамике число частиц $N$ сопряжено химическому потенциалу, то если число частиц неопределенно, то химический потенциал может быть задан точно, $\mu=0$ в предположении, что $\mu$ и $N$ проквантованы по Винеру и имеет место принцип неопределенности.

Противоречие между концепцией автора [12] и концепцией физиков, восходящей к Эйнштейну, также устраняется. В случае газа, для которого $N$ фиксировано согласно соотношению $\sum_{i=0}^{\infty} N_{i}=N$ [2], химический потенциал $\mu$ может быть малым положительным. Это очевидно, поскольку $N_{i} \leqslant N$, но это противоречит изначальной концепции Эйнштейна о том, что $\mu \leqslant 0$. Это противоречие устраняется, если для $\mu$ и $N$ справедливо соотношение принципа неопределенности, поскольку если $\mu=0$, то $N$ может принимать и бесконечные значения, а тогда $\mu>0$ невозможно.

Итак, можно сказать, что как гипотеза скейлинга, так и гипотеза винеровского квантования не согласуются в области критической точки со старой термодинамической концепцией четырех потенциалов. Однако гипотеза винеровского квантования не противоречит концепции четырех потенциалов, а дополняет ее, причем не только в критической точке, но и во всей области "газ-жидкость" согласуется с правилом Максвелла, а также устраняет логические неувязки в теории бозе-газа.

6 Теоретическая и математическая физика, т. 170, № 3, 2012 г. 
Отметим далее квантовый (имеется в виду винеровское квантование) скачок индекса в точках спинодали жидкой фазы. Классический индекс спинодали равен 2: $P \sim V^{2}$, аналогично точке поворота в квантовой механике, которой отвечает функция Эйри. Мы получаем аналогично (12) условия $\lim _{p \rightarrow 0} \frac{\widetilde{S}(p)}{p^{3}}<\infty,\left.\widetilde{S}^{(3)}(p)\right|_{p=0} \neq 0$.

При $x \rightarrow 0$ после замены $\xi / \sqrt[3]{\varepsilon}=y$ получаем $p_{\varepsilon}(x) \underset{x \rightarrow 0}{\longrightarrow} \sqrt[3]{\varepsilon} \cdot$ const. $\mathrm{B}$ нашем случае импульс $p_{\varepsilon}(x)$ есть объем $V$. Следовательно, аналогично рассуждению после формулы (12) получаем, что действие дробным оператором $D^{-1 / 3}$, примененным к единице, дает $\sim x^{1 / 3}, P \sim V^{3}$, и индекс в точках спинодали становится равным 3 .

ЗАмЕЧАНИЕ 3. Возможно, экспериментатор, рассматривая приближение критической изотермы при $T>T_{\text {cr }}$ к критической точке $\mu=0$, в силу принципа неопределенности сдвигается в сторону увеличения $N$, а значит, и увеличения плотности, и попадает на спинодаль жидкой фазы. Этот эффект аналогичен накапливанию гребня волны, который затем опрокидывается (часть частиц обгоняет точку возникновения ударной волны). Тогда критический индекс $\delta=4.3$ будет переходить в индекс спинодали $\delta=3$ (который случайно совпадает с классическим индексом критической точки). Этот переход, описываемый уравнением Власова, экспериментально замечен в [14] и других работах. Поэтому эксперименты Иванова [14] и Вагнера [15], [16], в которых при приближении к критической точке получены изменения критического индекса от $\delta=4.3$ до $\delta=3$, не противоречат нашей концепции.

В силу вышесказанного неопределенные и новые критические индексы возникают только благодаря квантованию сопряженных пар $\{P, V\},\{T, S\}$. Значит, соотношения между интенсивными величинами можно брать классические, поскольку предполагается бесконечно малая вязкость. В этом случае можно переходить и к другим координатам - давлению и плотности. Обозначив, как это принято, $p=\left(p-p_{\mathrm{cr}}\right) / p_{\mathrm{cr}}$, $T=\left(T-T_{\mathrm{cr}}\right) / T_{\mathrm{cr}}, v=\left(n-n_{\mathrm{cr}}\right) / n_{\mathrm{cr}}$, имеем в классическом случае [10] $p \sim v^{3}$, $v \sim \theta^{1 / 2}, \theta \sim v^{2}$. В классическом случае получаем $p \sim \theta^{3 / 2}$. В квантовом случае мы получим $\beta=0.375$ (ср. [13]). Принимая во внимание медленный рост значения $\mu\left(\rho, T_{\mathrm{cr}}\right)$ по отношению к $\rho$, мы получаем $\beta=0.350$. Это получается для проквантованного евклидовым образом уравнения ВДВ.

Из сказанного отнюдь не следует, что мы можем предъявить критические индексы, получаемые на эксперименте. Суть заключается в том, что при евклидовом квантовании поля параметр $\hbar$ - постоянная Планка - не зависит от координат и импульсов, а вязкость зависит. И реальная ударная волна в газе на самом фронте и в конце его имеет разную плотность, в отличие от ударной волны Хопфа для уравнения Бюргерса. Изменение величины $\varepsilon$ при переходе из газа в жидкость еще существенней.

Далее посмотрим на картину, которая у нас получилась для новой “непроквантованной” термодинамики [17]. На соответствующем графике критическая изотерма $T=T_{\text {сr }}$ совпадает с изохорой $P=P_{\text {сr }}$ на целом интервале значений $Z$ от $Z=0.444$ вплоть до $Z=0.17$, хотя, вообще говоря, совпадение должно быть только в точке $Z=0.296$. Это значит, что перегиб вида $v \sim p^{3}$ умножается на столь малый параметр, что точка перегиба растягивается.

Следовательно, малый параметр $\varepsilon$ участвует в этом растяжении, и если растяжение имеет порядок $\varepsilon^{1 / 3}$, то критический индекс $\delta$ будет в точности совпадать с экспериментальным значением. Это значит, что экспериментальное значение критического показателя помогает нам определить степень сжатия картины в плоскости $\{Z, P\}$ для $P_{\text {cr }}$ и $T_{\text {cr. }}$. Для модели ВДВ она значительно у́же. 
Классические соотношения между $T$ и $P$ дают теперь для модели ВДВ параметр $\beta=0.375$. Для $\mathrm{CO}_{2}$ и $\mathrm{Ar}$ за счет растяжения получаем $\beta=0.350$ (экспериментальное значение составляет $0.348 \pm 0.0013)$. Таким образом, здесь проявляется связь термодинамической картины с диффузией (вязкостью).

Благодарности. Работа выполнена при финансовой поддержке РФФИ (грант № 11-01-12058-офи_м).

\section{Список литературы}

[1] В. П. Маслов, ТМФ, 165:3 (2010), 543-567.

[2] Л. Д. Ландау, Е. М. Лифшиц, Теоретическая физика, т. 5: Статистическая физика, Наука, М., 1976.

[3] E. M. Apfel'baum, V.S. Vorob'ev, Russ. J. Math. Phys., 18:1 (2011), 26-32.

[4] В. П. Маслов, ТМФ, 101:3 (1994), 433-441.

[5] В.П. Маслов, Квантование термодинамики и ультравторичное квантование, ИКИ, M., 2001.

[6] V.P. Maslov, Russ. J. Math. Phys., 18:4 (2011), 440-464.

[7] Б. Ван дер Поль, Х. Бреммер, Операчионное исчисление на основе двустороннего преобразования Лапласа, ИЛ, М., 1952.

[8] Л. Д. Ландау, Е. М. Лифшиц, Теоретическая физика, т. 3: Квантовая механика (нерелятивистская теория), Наука, М., 1974.

[9] В. П. Маслов, УМН, 38:6(234) (1983), 3-36.

[10] M.S. Green, "Introduction", Proceedings of the Conference on Phenomena in the Neighborhood of Critical Points, NBS Misc. Publ., 273, eds. M. S. Green, J. V. Sengers, NBS, Washington, 1966, ix-xi.

[11] Д. Ю. Иванов, Вестн. СибГУТИ, 3 (2009), 94-104.

[12] V.P. Maslov, Russ. J. Math. Phys., 18:4 (2011), 440-464, arXiv: 1111.6106.

[13] Р. Балеску, Равновесная и неравновесная статистическая механика, 1, Мир, М., 1978.

[14] Д. Ю. Иванов, Критическое поведение неидеализированных систем, Физматлит, М., 2003.

[15] W. Wagner, N. Kurzeja, B. Pieperbeck, Fluid Phase Equilibria, 79 (1992), 151-174.

[16] N. Kurzeja, Th. Tielkes, W. Wagner, Int. J. Thermophys., 20:2 (1999), 531-562.

[17] V.P. Maslov, Math. Notes, 90:3-4 (2011), 533-547.

Поступила в редакцию 30.10 .2011 